\title{
Guía Clínica de VIH/SIDA
}

\author{
Alejandro Afani S., Carlos Pérez C., Patricia Vásquez T. y Marcelo Wolff R.
}

\author{
Guidelines on HIV/AIDS
}

$\mathrm{E}$ n este número de Revista Chilena de Infectología se publican las Guías Clínicas de VIH/SIDA. Este es el fruto de un arduo trabajo de muchos meses de un grupo de especialistas con un directo quehacer en la atención de pacientes infectados por VIH. Estas guías fueron presentadas hace meses al Ministerio de Salud y se oficializaron a principios de 2010. Los objetivos centrales de la guía son dos:

- Detener la progresión de la enfermedad por VIH, disminuir la morbi-mortalidad por SIDA y enfermedades asociadas y mejorar la calidad de vida de los adultos infectados por VIH, mediante el acceso universal y oportuno a tratamiento con asociaciones de antiretrovirales (ARV) de eficacia, durabilidad y seguridad probadas.

- Disminuir la tasa de transmisión vertical del VIH a menos de $2 \%$ global y a $1 \%$ o menos en los binomios madre-hijo que reciben protocolo completo, mediante la oferta universal del test de VIH en la mujer embarazada con consejería previa y la aplicación de medidas farmacológicas y no farmacológicas de eficacia probada en la reducción de la transmisión, sin afectar las posibilidades terapéuticas futuras de la madre y del hijo.

Un objetivo adicional de la presente guía era actualizar la terapia antiretroviral (TAR) en el país que, a pesar de haber sido pionero, se había quedado atrás incluso a lo recomendado por la Organización Mundial de la Salud. Esta actualización se ha dado tanto en la indicación de inicio de terapia, llevándola a etapas más precoces, donde ya se había demostrado su superioridad, incluyendo nuevas situaciones donde es necesario iniciar la terapia e incorporar las muy necesarias nuevos fármacos ARV.

Creemos que el resultado final de esta guías resuelve, en gran medida, estas nuevas necesidades y para un país con un sistema de autorización y otorgamiento de la TAR tan centralizado, constituye un gran respaldo para la aplicación por parte de los equipos tratantes de los mejores estándares de atención posible.

Son muchas las evidencias que hacen aconsejable el inicio más precoz de TAR, por lo que se estableció empezarla con un recuento de linfocitos TCD4 de 350 por $\mathrm{mm}^{3}$ o menos en pacientes asintomáticos adultos. Se hacen consideraciones especiales en ciertas patologías como trombocitopenia o nefropatía asociada a VIH en que no se considera el recuento de CD4 como criterio de inicio.

Las ventajas de iniciar antes la terapia se basa en numerosos estudios, pero uno de los más sólidos en fortaleza científica es el sub-análisis de los pacientes que no empezaron TAR en el estudio SMART, en que se demostró 3,5 veces más eventos (infecciones oportunistas y eventos serios no definitorios de SIDA, especialmente cardiovasculares) que los que la iniciaron ${ }^{1}$.

Por otra parte, el estudio NA-ACCORD muestra un riesgo aumentado de morir con un inicio tardío de TAR². La recuperación inmunológica que se logra empezando la TAR antes es avalada también en estudios nacionales ${ }^{3}$ De acuerdo a la evidencia el impacto a más largo plazo significará una reducción en el riesgo cardiovascular ${ }^{4}$ y la aparición de neoplasias ${ }^{5}$. Sin duda, el acceso ampliado y oportuno a TAR también tiene un impacto en la transmisión como se ha demostrado en trabajos, tanto en parejas discordantes ${ }^{6}$ como a nivel poblacional $^{7}$. La guía establece también una recomendación terapéutica adecuada al problema de la co infección de VIH y hepatitis B, que estaba recibiendo un tratamiento subestándar hasta ahora.

En el capítulo de prevención de transmisión vertical (TV) se incluye recomendaciones del control de la mujer embarazada con infección por VIH y los distintos escenarios a los que se pueden ver enfrentados los médicos tratantes.

Está claramente establecido que el factor independiente más importante que aumenta el riesgo de TV es la carga viral por lo que el diagnóstico oportuno en el embarazo de la condición de infectada por VIH y el inicio de la TAR con tres ARVs, a más tardar en la semana 24 de gestación, permite reducir el riesgo de TV a $2 \%$ o menos. Tomando todas las medidas, tanto de diagnóstico precoz, control, tratamiento, resolución del parto y suspensión de la lactancia materna, se debería lograr reducir la TV a menos de $1 \%$ en nuestro país y llegar a una situación en que los casos de infección por VIH en niños sean excepcionales.

Se considera que en un panorama ideal, las guías debieran actualizarse al menos anualmente en una búsqueda por ofrecer a los pacientes las mejores opciones para su tratamiento, así como una herramienta que permita a
Comisión Asesora de CONASIDA. 
los médicos tratantes mejorar su cometido acorde a las nuevas evidencias. Para cumplir este objetivo se requiere un compromiso de las autoridades. Este compromiso ha sido claramente insuficiente, así como lo ha sido el poco interés demostrado en implementar la oferta amplia del test con una consejería simple y funcional que permita el tratamiento oportuno que nuestros pacientes merecen. Esperamos que esta deuda que tienen las autoridades sa- nitarias con la población infectada y los equipos tratantes se resuelva rápidamente y no se vuelva a acumular en el futuro.

Creemos que esta guía será de mucha utilidad en su labor diaria y los convocamos a colaborar con nosotros en su adecuada implementación, como también a optimizar el diagnóstico temprano de la infección por VIH, a la luz de los enormes beneficios que implica su tratamiento.

\section{Referencias}

1.- Emery S, et al. Major clinical outcomes in antiretroviral therapy (ART)-naive participants and in those not receiving ART at baseline in the SMART study. J Infect Dis 2008; 197: 1133-44.

2.- Kitahata MM, et al. Effect of early versus deferred antiretroviral therapy for HIV on survival N Engl J Med 2009; 360: 1815-26.

3.- Cortés C, Beltrán C, Muñoz R, Daube E, Wolff $\mathrm{M}$ por el grupo SIDA Chile: Impacto de la inmunodepresión basal y su grado de recuperación al año de terapia antirretroviral en sobrevida, complicaciones oportunistas y reacción de recuperación inmune. Rev Med Chile 2008; 136: 1503-10.

4. Monforte A, Abrams D, Pradier C, Weber R, Reiss $\mathrm{P}$, Bonnet $\mathrm{F}$, et al for the Data Collection on Adverse Events of Anti-HIV Drugs (D:A:D) Study Group. HIV-induced immunodeficiency and mortality from AIDS-defining and nonAIDS-defining malignancies. AIDS 2008; 22 : 2143-53.

5. Phillips A, Carr A, Neuhaus J, Visnegarwala F, Prineas R, Burman W, et al. Interruption of antiretroviral therapy and risk of cardiovascular disease in persons with HIV-1 infection: exploratory analyses from the SMART trial. Antivir Ther 2008; 13: 177-87.

6. Donnell D, Kiarie J, Thomas K, Baeten J, Lingappa J, Cohen C, et al. ART and risk of heterosexual HIV-1 transmission in HIV-1 serodiscordant African couples: A multinational prospective study. CROI 2010. Sn Fco-Ca-EUA. Abstract 136

7. Montaner J, Wood E, Kerr T, Yip B, Lima V, Shannon K, et al. Association of expanded HAART coverage with a decrease in new HIV diagnoses, particularly among injection drug users in British Columbia, Canada CROI 2010 Sn Fco-Ca-EUA. Abstract 88LB. 